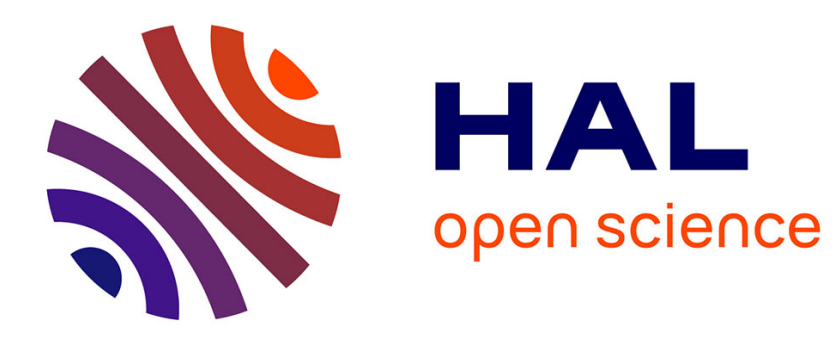

\title{
Long Elastic Open Neck Acoustic Resonator for low frequency absorption
}

Frank Simon

\section{To cite this version:}

Frank Simon. Long Elastic Open Neck Acoustic Resonator for low frequency absorption. Journal of Sound and Vibration, 2018, 421, pp.1-16. 10.1016/j.jsv.2018.01.044 . hal-01870188

\section{HAL Id: hal-01870188 \\ https://hal.science/hal-01870188}

Submitted on 7 Sep 2018

HAL is a multi-disciplinary open access archive for the deposit and dissemination of scientific research documents, whether they are published or not. The documents may come from teaching and research institutions in France or abroad, or from public or private research centers.
L'archive ouverte pluridisciplinaire HAL, est destinée au dépôt et à la diffusion de documents scientifiques de niveau recherche, publiés ou non, émanant des établissements d'enseignement et de recherche français ou étrangers, des laboratoires publics ou privés. 


\title{
1 Long Elastic Open Neck Acoustic Resonator for low frequency absorption
}

Frank Simon

Onera Centre de Toulouse, 2 avenue Edouard Belin, 30155, Toulouse, France

Corresponding author: Frank Simon: frank.simon@onera.fr

\begin{abstract}
Passive acoustic liners, used in aeronautic engine nacelles to reduce radiated fan noise, have a quarter-wavelength behavior, because of perforated sheets backed by honeycombs (with one or two degrees of freedom). However, their acoustic absorption ability is naturally limited to medium and high frequencies because of constraints in thickness. The low ratio "plate thickness/hole diameter" generates impedance levels dependent on the incident sound pressure level and the grazing mean flow (by a mechanism of nonlinear dissipation through vortex shedding), which penalises the optimal design of liners. The aim of this paper is to overcom this problem by a concept called LEONAR ("Long Elastic Open Neck Acoustic Resonator"), in which a perforated plate is coupled with tubes of variable lengths inserted in a limited volume of a back cavity. To do this, experimental and theoretical studies, using different types of liners (material nature, hole diameter, tube length, cavity thickness) are described in this paper. It is shown that the impedance can be precisely determined with an analytical approach based on parallel transfer matrices of tubes coupled to the cavity. Moreover, the introduction of tubes in a cavity of a conventional resonator generates a significant shift in the frequency range of absorption towards lower frequencies or allows a reduction of cavity thickness. The impedance is practically independent of sound pressure level because of a high ratio "tube length/tube hole diameter". Finally, a test led in an aeroacoustic bench suggests that a grazing flow at a bulk Mach number of 0.3 has little impact on the impedance value. These first results allow considering these resonators with linear behavior as an alternative to classical resonators, in particular, as needed for future Ultra High Bypass Ratio engines with shorter and thinner nacelles.
\end{abstract}

\section{Keywords:}

Sound insulation, Transmission resonator, Acoustic impedance, Local reaction, Absorbing material 


\section{Introduction}

Locally reacting liners, as those used in aeronautical engine nacelles, are generally "sandwich" resonators with a perforated plate linked to an honeycomb material above a rigid plate. Their absorption behavior can be described approximately with the principle of a Helmholtz resonator. The frequency range of absorption is thus essentially controlled by the thickness of the honeycomb cavity ("quarter-wavelength" behavior). Honeycomb cells are necessary to force wave direction perpendicularly to the perforated plate. It is classical to verify that the cell diameter $d$ is lower than the minimal half wavelength. Moreover, the cell layers are supposed to be rigid (no vibration or damping). The small size of the holes (mostly from 0.4 to $2 \mathrm{~mm}$ according to industrial needs), absorbs the energy (through the acoustic boundary layer applied at the internal walls) when a wave is propagated through the resonant cavity [1,2]. The impedance can depend non-linearly on the incident particle velocity level (or sound pressure level) [1]) and on the grazing flow. Acoustic "vortices" of particle velocity can therein occur at the resonator surface, thus modifying impedance. Many studies, since Ingard in the 1950s [3], have tried to determine the influence of various parameters on the impedance and the absorption of holes. Gaeta and Ahuja [4] show in particular that to increase the perimeter of the hole with the same surface allows an increase of the absorption at low magnitudes of particle velocity $(<1$ $\mathrm{ms}^{-1}$ ) but has no significant effect for higher velocities. Above a threshold value of the ratio " $v o / v *$ " (acoustic or particle velocity/friction velocity of the acoustic boundary layer) the hole behavior becomes non-linear [5]. It appears that the nonlinear dissipation mechanism of vortex shedding is crucial for noise levels greater than $120 \mathrm{~dB}$ [6], values unfortunately much lower than in an aircraft engine. Chandrasekharan et al. [7] led impedance measurements in a tube and compared results with classical laws of Hersh, Kraft and Candrall \& Melling. It is shown that an increase of ratio " $l_{p} / r$ " (plate thickness/hole radius) increases the frequency band over which there is linear behavior of the plate with the sound level (between 100 and $150 \mathrm{~dB}$ until 6,4 kHz). Boden et al. [8] show that, for a high pressure level noise at different tones, to modify the sound pressure level at one particular frequency can generate a non-linear variation of impedance at other frequencies. Indeed, if the acoustic excitation is periodic with multiple harmonics, the impedance at a given frequency may depend on the particle velocity at other frequencies. It is proven that a simply linear model of the impedance cannot be enough to accurately characterize a material. That is the reason why it can be interesting to determine an impedance model taking into account the non-linearity and to then separate linear and non-linear parts [9]. In [10] the ratio " $v o / v *$ " is also introduced as a parameter (similar to [5] for a single resonator) to determine a limit of linearity that depends on the Mach Number: 1

41 for Mach 0.15, 0.3 for Mach 0.3 and 0.1 for Mach 0.51. The grazing flow 42 produces non-linearity phenomena for lower acoustic velocities. In [11], it is 
specifed that the resistance of several samples (liner diameter $140 \mathrm{~mm}$, liner thickness $1 \mathrm{~mm}$, hole diameter $1.25 \mathrm{~mm}$, porosity 0.42 and $6.79 \%$ ) increases with the Mach Number (Mach $0-0.1$ at 1,400 Hz). Jones et al. [12] have tried to identify the influence of hole diameter with a grazing flow $(0 \leq M \leq 0.5)$, and with a variation of porosity $(6.4 \% \leq \sigma \leq 13.2 \%)$, plate thickness $\left(0.51 \mathrm{~mm} \leq l_{p} \leq 1.02 \mathrm{~mm}\right), l_{p} / d\left(0.34 \leq l_{p} / d \leq 0.80\right)$ and cavity length $h$ $7 \quad(38.1 \mathrm{~mm} \leq h \leq 76.2 \mathrm{~mm})$. It appears that $l_{p} / d$ has no influence on the 8 impedance up to Mach 0.3. Beyond this Mach number, the resistance tends to 9 increase slightly, especially for low values of $l_{p} / d$. The resonance frequency 10 decreases obviously as $h$ increases. In $[13,14]$, in order to reduce the frequency 11 band of the absorption, the concept of a material with straight main pores 12 bearing lateral cavities (dead-ends) is studied. The presence of dead-ends 13 significantly alters the acoustical properties of the material and can significantly 14 increase the absorption at low frequencies, because of a low sound speed in the 15 main pores and thermal losses in the dead-end pores.

Finally, in order to enlarge the frequency range of absorption, different types of one degree of freedom liners can be stacked to constitute two or three degrees of freedom liners. In such cases, the increase of sound pressure level increases their resistance and decreases their reactance [15]. The presence of grazing flow even seems to increase the resistance and to have no influence on the reactance. Furthermore, it would generate strong sound levels for Strouhal Number (defined with regard to the grazing flow) from 0.1 to 0.4 . The authors develop a non-linear model of impedance based on Helmholtz formulations, in uniform grazing flow: the non linear terms are only relative to the first cavity. Nevertheless, the physical law of two or three degrees of freedom liners is not suited to an absorption at the lowest frequencies, as needed for future Ultra High Bypass Ratio (UHBR) engines with shorter and thinner nacelles (frequencies around $500 \mathrm{~Hz}$ ).

A possible approach could be to include, in a Helmholtz resonator, a winding neck extension built at the upper surface for tuning at a low frequency [16], or to link an upper perforated panel with flexible tubes introduced in the cavity, as proposed by Lu et al. [17].

In these configurations, incident acoustic waves are damped in a long resistive and reactive medium (winding neck extension [16] or flexible tubes [17]) before being transmitted in the cavity. The interface with the cavity generates a low resonance frequency by a prolongation of the air column length (end correction of the hole neck). Indeed, the analogy with Helmholtz resonator shows that, in the case of long tubes, the resonance frequency can be governed 39 by the tube length $l_{\text {tube }}$ with an effect comparable to cavity thickness $h$ 
1 (frequency dependence in $\frac{1}{\sqrt{\left(l_{\text {neck }}+l_{\text {tube }}\right) h}} \approx \frac{1}{\sqrt{l_{\text {tube }} h}}$, the perforated plate

thickness being negligible compared to the tube length).

The interest of this concept has been proven experimentally by these last authors but without any mathematical model to allow for determination of the absorption frequency range according to dimensional parameters.

The aim of this paper is therefore, firstly, to implement a mathematical model without the hypothesis of a short tube, in order to describe a concept of a perforated plate coupled with tubes of variable lengths that fill a limited volume of a cavity (LEONAR for "Long Elastic Open Neck Acoustic Resonator"), then to validate this concept with materials having one or several lengths of flexible tubes within different cavities. The potentialities of additive manufacturing, as shown for example by Setaki et al. for combination of multiple resonators [18], can also be used in order to manufacture plates with tubes and cavity cells in the same process without classical problems of gluing. Indeed, the liner manufacturing is generally carried out in two stages: the process begins firstly by laser drilling of an upper thin plate to generate the desired porosity, and continues secondly by the bonding of this plate on the honeycomb. With this fabrication method, the glue can fill holes that are too close to the honeycomb cells, a problem avoided by $3 \mathrm{D}$ printing by additive manufacturing with Selective Laser Sintering or Stereolithography for polymers and Selective laser Melting for metal.

Some configurations are thus simulated and tested to evaluate the relevance of theoretical approach and the linearity of behavior vs. incident Sound Pressure Level. The impedance of such a material is also determined in a duct with a high grazing flow (Mach number 0.3) for comparison with its impedance without flow.

This paper completes previous author's communications $[19,20]$.

\section{Description of LEONAR}

The resonator is composed of a perforated plate whose holes are connected to hollow flexible or rigid tubes, inserted in a cavity ended by a rigid wall (Fig. 1).

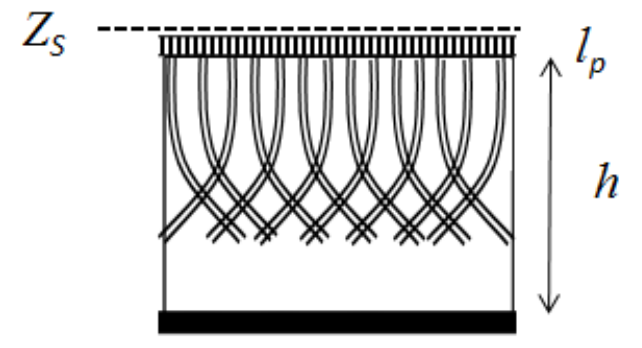

Fig. 1. Illustration of resonator with an upper perforated plate (thickness $l_{p}$ ) connected to hollow flexible tubes inserted in a cavity (thickness $h$ ). 
1 The parameters describing the resonator are, respectively: thickness $l_{p}$ and 2 porosity $\sigma_{p}$ of plate, inner radius $r_{i}$ and outer radius $r_{o}$ of the tubes, tube length $l_{t}$ 3 and cavity thickness $h . \sigma_{p}$ is defined as the ratio of the area of holes to the total 4 area of plate.

5 Examples of resonators (with tubes in Teflon or in PMMA for PolyMethyl 6 MethAcrylate, also known as Acrylic) are shown below (Fig. 2 to 5).

Fig. 2. Type 1 samples with a perforated plate connected to Teflon tubes of variable lengths, to be placed above a cavity.

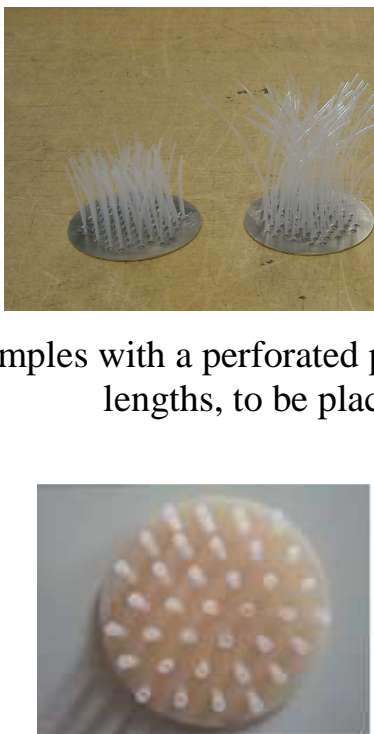

(a)

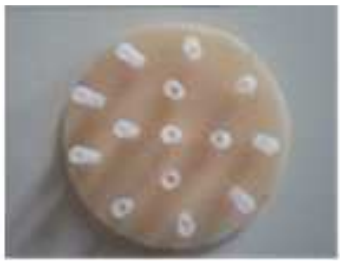

(b)

14 Fig. 3. Type 2 samples with a perforated plate connected to PMMA tubes of variable radius (a) and (b), to be placed above a cavity.

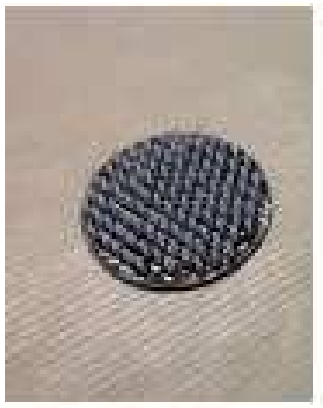

(a)

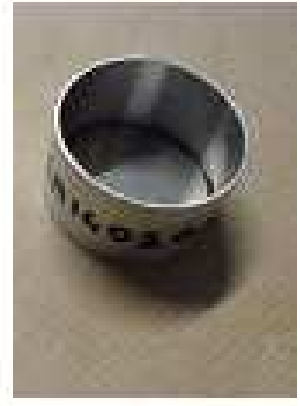

(b)

Fig. 4. Type 3 samples with a perforated plate connected to tubes (a) to be placed above a cavity ended by a rigid wall (b). 


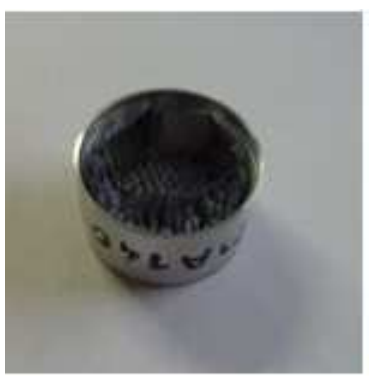

(a)

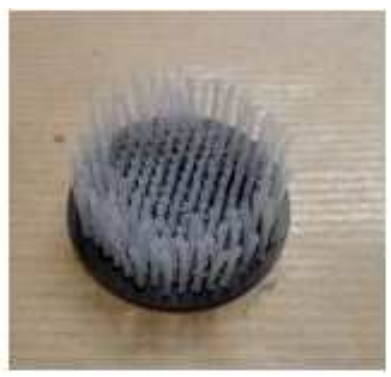

(b)

3 Fig. 5. Type 3 samples with a perforated plate connected to tubes of 2 variable lengths, partitioned (a) or not (b), to place above a cavity.

The propagation of waves along hollow tubes can be shown as a linear combination of propagational, thermal and viscous modes [21]. The pressure in the propagational mode satisfies an ordinary wave equation, while the temperature and velocity in the other modes satisfy diffusion equations.

To simplify the mathematical description, sound propagation is considered in narrow channels between plane parallel plates separated by a distance $\left(2 r_{i}\right)$.

The pressure field $p$ is mainly due to the propagational mode, for harmonic time dependence (expressed by $\omega$ ), satisfies the classical equation:

$$
\nabla^{2} p+\left(\frac{\omega}{c}\right)^{2} p=0
$$

which has solutions in the form

$$
p(x, r)=A \cos \left(q_{r} r\right)\left(e^{\mathrm{j} q x}+e^{-\mathrm{j} q x}\right)
$$

with $r=0$ at the center, $r= \pm r_{i}$ at the boundaries, $q_{r}$ and $q$ being, respectively the complex transverse and axial wavenumbers, related by:

$$
q^{2}=\left(\frac{\omega}{c}\right)^{2}-q_{r}^{2}
$$

We assume that the wavelength of waves $\lambda$ is much larger than $2 r_{i}$ (i.e. $\lambda>137$ $\mathrm{mm}$ up to $2500 \mathrm{~Hz}$ (at ambient air conditions), compared to $1 \mathrm{~mm}$ for typical liner hole diameter) and therefore without visco-thermal effects, $q_{r}=0$. On the other hand, to account for these effects consists in determining $q_{r} \neq 0$ from the wall boundary conditions of zero total tangential velocity and zero temperature fluctuation. Thus, $q_{r}$, parameter common to all modes, can be inferred from the
31 mathematical expression of transverse velocities due to propagational, thermal 
7 - $k_{v}=\frac{1+\mathrm{j}}{\delta_{v}}$ where $\delta_{v}=\sqrt{\frac{2 \mu}{\rho \omega}}$ (viscous boundary layer thickness)

and viscous modes. Indeed, as the sum of transverse velocities must vanish at the inner boundaries, the transverse propagation constant (with the assumption of narrow channels: $q_{r} r_{i}<<1$ or $\left.\cos \left(q_{r} r_{i}\right) \approx 1\right)$ is given by [21]:

$$
q_{r}^{2}=-\left(\frac{\omega}{c}\right)^{2} \frac{(\gamma-1) F\left(k_{h} r_{i}\right)+F\left(k_{v} r_{i}\right)}{1-F\left(k_{v} r_{i}\right)} \text { where } F(X)=\frac{\tan (X)}{X}
$$

with

- $k_{h}=\frac{1+\mathrm{j}}{\delta_{h}}$ where $\delta_{h}=\sqrt{\frac{2 K}{\rho C_{p} \omega}}$ (thermal boundary layer thickness)

$c, K, C_{p}, \rho$ and $\mu$, are respectively sound speed, heat conduction coefficient, specific heat at constant pressure, density and dynamic viscosity.

In narrow channels, the average axial velocity has the following form:

$$
u_{x}=\frac{A q}{\omega \rho}\left(1-F\left(k_{v} r_{i}\right)\right)\left(e^{\mathrm{j} q x}-e^{-\mathrm{j} q x}\right)
$$

Finally, the complex propagation constant in the axial direction of the channel $q$ is determined simply by [21] :

$$
q=\left(\frac{\omega}{c}\right) \sqrt{\frac{1+(\gamma-1) F\left(k_{h} r_{i}\right)}{1-F\left(k_{v} r_{i}\right)}}
$$

For circular tubes, the function $F(X)=\frac{\tan (X)}{X}$, introduced in Eqs. (4), (7) and (8) to determine the transverse and axial propagation constants, are generally replaced by Bessel functions [26]. In low frequency, one can nevertheless keep this function and replace $r_{i}$ by $r_{i} / 2$ in these equations, without significant differences in the configurations of resonators.

Subsequently, we assume that (Fig. 6):

- continuity of pressure and mass flow between the tubes and surrounding cavity is verified at the end of tubes,

- transmitted waves propagate in rigid cavity, without any loss, mainly in the direction of the thickness, as for a classical resonator. 


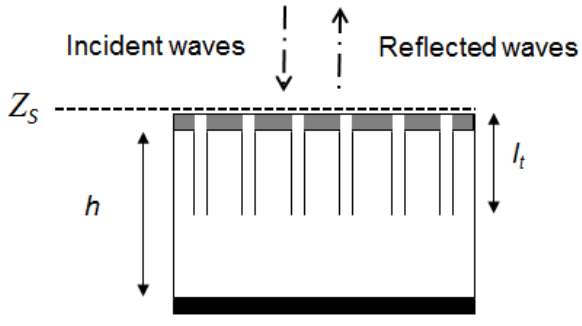

(a)

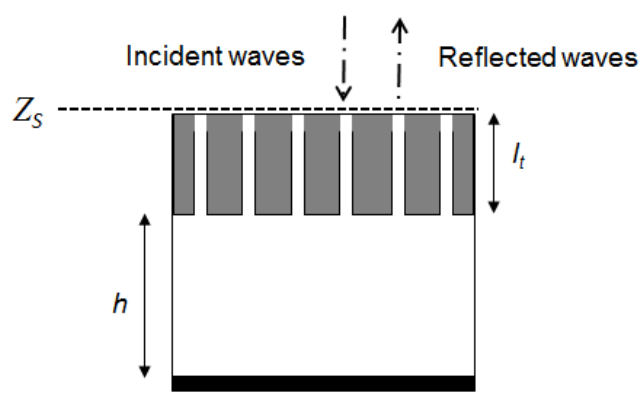

(b)

Fig. 6. Physical configuration (a) with an upper perforated plate connected to hollow tubes (length $l_{t}$ ) inserted in a cavity (thickness $h$ ) - Simulated configuration (b) with an equivalent thick perforated plate (thickness $l_{t}$ ) inserted in a cavity (thickness $h$ ).

One can easily define the different transfer matrices related to elementary components (Fig. 6 (b)), as follows:

9

10

11

12

The specific impedance at the structure surface (Fig. 6) is given by the plate porosity $\sigma_{p}$ and normalized by the fluid impedance $\rho c$ :

$$
\frac{Z_{s}}{\rho c}=-\left(\frac{T_{11}}{T_{21}}\right)^{*} \frac{1}{\rho c \sigma_{p}}
$$

Classical inductive and resistive end corrections can also be added for a specific impedance, i.e., [22], but the effects are relatively negligible for long tubes and low frequencies.

One can also extend the concept with configurations comprising simultaneously comprised of several tube lengths in different cavities (with a 
partition) or in the same cavity (without partition) (Fig. 7). One considers, as an 2 example, 2 tube lengths (tubes " 1 " and " 2 ") with the same hole distribution (i.e.

3 a porosity $\sigma_{p}$ ):

4

- with partition (Fig. 7 (a)), for which tubes "1" and "2" are in different cavities:

The global specific impedance is then derived from the individual specific impedance Eq. (13) of each tube group (cells with tubes "1" or " 2 "), by an admittance average:

$$
\frac{Z_{\text {s partition }}}{\rho c}=\frac{2}{\rho c\left(\frac{1}{Z_{s 1}}+\frac{1}{Z_{s 2}}\right)}
$$

- without a partition (Fig. 7 (b)), for which tubes "1" and "2" are in the same cavity:

18 individual tube transfer matrices $\mathbf{T}_{\text {tube } \mathrm{m}}$ (Eq. (10)), as follows:

19

$$
\mathbf{Y}_{\text {tube }}=\mathbf{Y}_{\text {tube 1 }}+\mathbf{Y}_{\text {tube 2 }}
$$

22 with $\mathbf{Y}_{\text {tube } \mathrm{m}}=\left[\begin{array}{cc}\frac{T_{\text {tube 22 }}}{T_{\text {tube 12 }}} & \frac{T_{\text {tube 12 }} \cdot T_{\text {tube 21 }}-T_{\text {tube 11 }} \cdot T_{\text {tube 22 }}}{T_{\text {tube 12 }}} \\ \frac{1}{T_{\text {tube 12 }}} & -\frac{T_{\text {tube 11 }}}{T_{\text {tube 12 }}}\end{array}\right]_{i}$ for $m \in\{1,2\}$,

24 then to compute $\mathbf{T}_{\text {tubes/cavity }}$, the global transfer matrix $\mathbf{T}_{\text {tube }}$ from Eq. (15) and $25 \mathbf{T}_{\text {total }}$ from Eq. (12):

26

$$
\begin{aligned}
& \mathbf{T}_{\text {tubes/cavity }}=\left[\begin{array}{cc}
1 & 0 \\
0 & 2 / \sigma_{p}
\end{array}\right] \\
& \mathbf{T}_{\text {tube }}=\left[\begin{array}{cc}
-\frac{Y_{\text {tube 22 }}}{Y_{\text {tube 12 }}} & \frac{1}{Y_{\text {tube 12 }}} \\
\frac{Y_{\text {tube 12. }} \cdot Y_{\text {tube 21 }}-Y_{\text {tube 11 }} \cdot Y_{\text {tube 22 }}}{Y_{\text {tube 12 }}} & \frac{Y_{\text {tube 11 }}}{Y_{\text {tube 12 }}}
\end{array}\right]_{i}
\end{aligned}
$$


The impedance normalized to $\rho c$ is expressed by:

$$
\frac{Z_{\mathrm{s} \text { no partition }}}{\rho c}=-\left(\frac{T_{11}}{T_{21}}\right)^{*} \frac{2}{\rho c \sigma_{p}}
$$

Finally, the reflection and absorption coefficients, respectively $R$ and $\alpha$, relative to a normal incident excitation are expressed by:

$$
R=\frac{Z_{S}-\rho c}{Z_{S}+\rho c}
$$

from specific impedances (Eqs. (13), (14) and (19)).

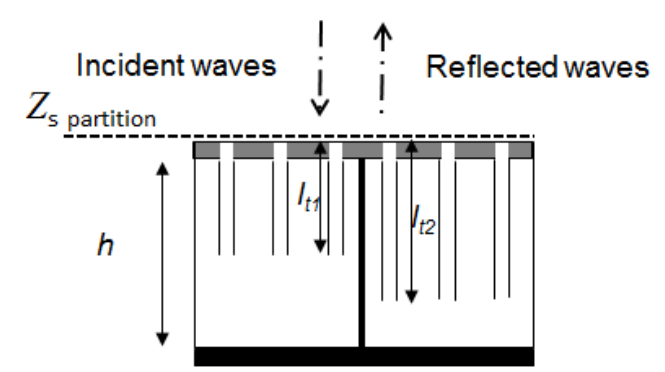

(a)

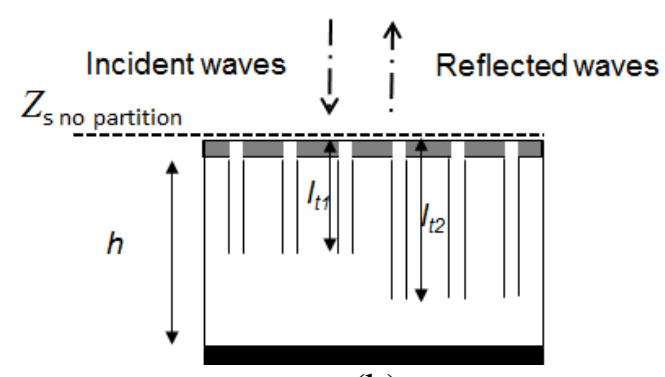

(b)

Fig. 7. Physical configurations with an upper perforated plate connected to two types of hollow tubes (lengths $l_{t l}$ and $l_{t 2}$ ) inserted in two cavities (a) or in the same cavity (b).

\section{Validation}

The validation is led in an impedance tube on 3 types of resonator for which the tubes are flexible or rigid, with variable tube radii and lengths and with or without rear partition.

A test is also conducted in presence of a high grazing flow for comparison.

The characteristics are specified below for types 1 and 2 . However for confidentiality, absolute values cannot be given for type 3 and only relative values are indicated.

\subsection{Type 1 and 2 Resonator}

The impedance is obtained in an impedance tube instrumented with 3 microphones for pressure measurements. On the opposite side of the tube, the loudspeaker generates a broadband random noise propagated in plane waves 
from 100 to $2,500 \mathrm{~Hz}$ and from 100 to $145 \mathrm{~dB}$. The standard measurement 2 method for two microphones is used in accordance with references [23-25]. The 3 three microphones taken by pair allow satisfying the total frequency range.

First tests have been conducted for type 1 samples whose characteristics are specified in Table 1.

\section{Table 1}

Characteristics of type 1 samples.

\begin{tabular}{ccccccc}
\hline $\begin{array}{c}l_{p} \\
(\mathrm{~mm})\end{array}$ & $\begin{array}{c}\sigma_{p} \\
(\%)\end{array}$ & $\begin{array}{c}r_{i} \\
(\mathrm{~mm})\end{array}$ & $\begin{array}{c}r_{o} \\
(\mathrm{~mm})\end{array}$ & $\begin{array}{c}l_{t} \\
(\mathrm{~mm})\end{array}$ & $\begin{array}{c}h \\
(\mathrm{~mm})\end{array}$ & $l_{t} / r_{i}$ \\
\hline 1 & 1.92 & 0.35 & 0.55 & 10 & 35.9 & 28 \\
& & & & 20 & & 57 \\
& & & & 30 & & 86 \\
& & & & 60 & & 171 \\
& & & 90 & & 257 \\
\hline
\end{tabular}

It appears that these materials have a linear behavior independent of the incident acoustic pressure level, which is representative of a constant impedance and absorption coefficient (ex. for sample with $l_{t}=20 \mathrm{~mm}$ in Fig. 8), while a sample with only the perforated plate (without tubes) generates a large variation of absorption (Fig. 9). Non-linearity is due to acoustic vortices around the holes 20 for a low ratio of " $l_{p} / r_{i}$ " [6,7]. Therefore, artificially increasing the plate 21 thickness, by extending the tubes, prevents the presence of vortices. In the 22 present case, the ratio is between 28 to 257 for type 1 samples, compared with 231.8 for the perforated plate, which guarantees the linear behavior regardless the 24 excitation configuration.

One can notice, also, that the frequency range of absorption is very different: around $260 \mathrm{~Hz}$, for the resonator with tubes, vs. $1,300 \mathrm{~Hz}$ for the classical resonator. On the other hand, the rises in medium frequency range are not controlled. The comparison of absorption coefficient for all samples (Fig. 10) confirms that the length of the tube allows shifting the frequency range of 30 absorption (resonator thickness lower than $\lambda / 30$ ). Nevertheless, an increase in 31 length can be associated with a reduction of absorption coefficient, i.e. if $l_{t}>h$,

32 essentially because of a significant reduction of cavity volume. 


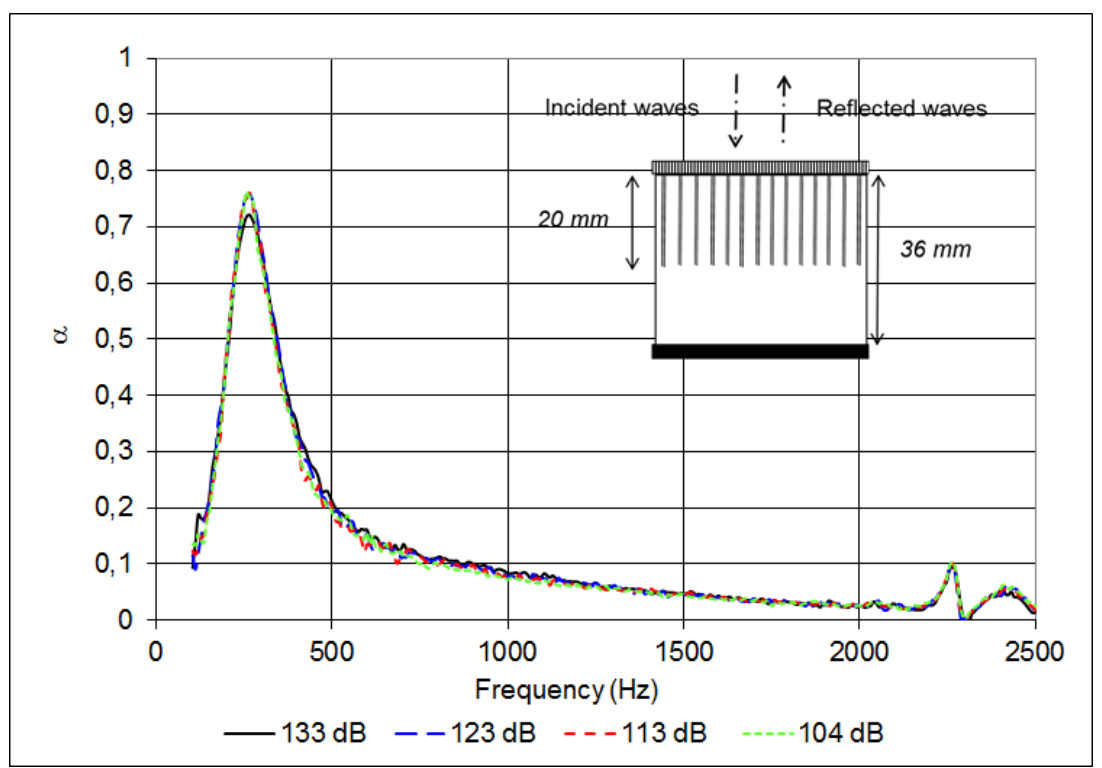

Fig. 8. Effect of Soud Pressure Level (dB) on absorption coefficient for a type 1 sample with $l_{t}=20 \mathrm{~mm}$ and $h=35.9 \mathrm{~mm}$.

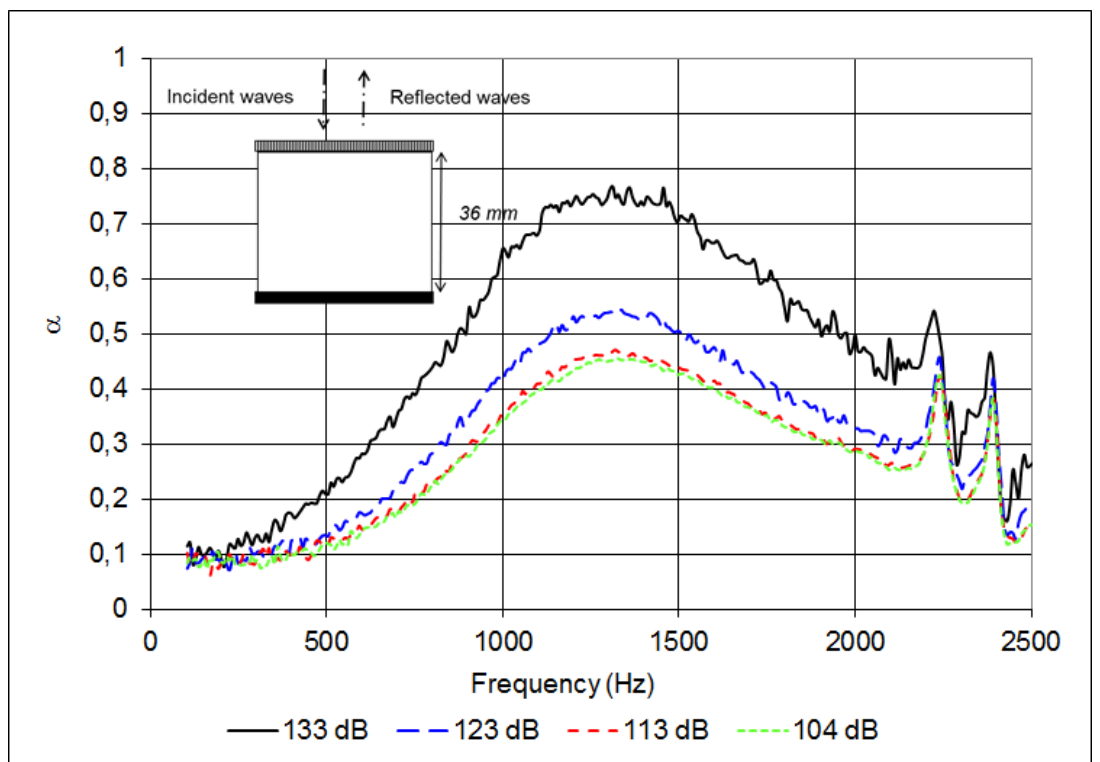

Fig. 9. Effect of Soud Pressure Level (dB) on absorption coefficient for a classical resonator with a thin perforated plate $\left(\sigma_{p}=4.7 \%, r_{o}=0.55 \mathrm{~mm}, l_{p}=1 \mathrm{~mm}\right)$ above a cavity of $35.9 \mathrm{~mm}$ thickness.

Simulations of reactance are led using Eq. (13) for several samples. The satisfying comparison with experimental results gives more confidence in determining the frequency range of absorption, relative to "0" reactance (Fig. 
1 11), compared to the classical Helmholtz formulation or Lu et al. [17] approach 2 applied to long tubes.

4 Fig. 10. Effect of tube length (between 10 to $90 \mathrm{~mm}$ ) on absorption coefficient for type

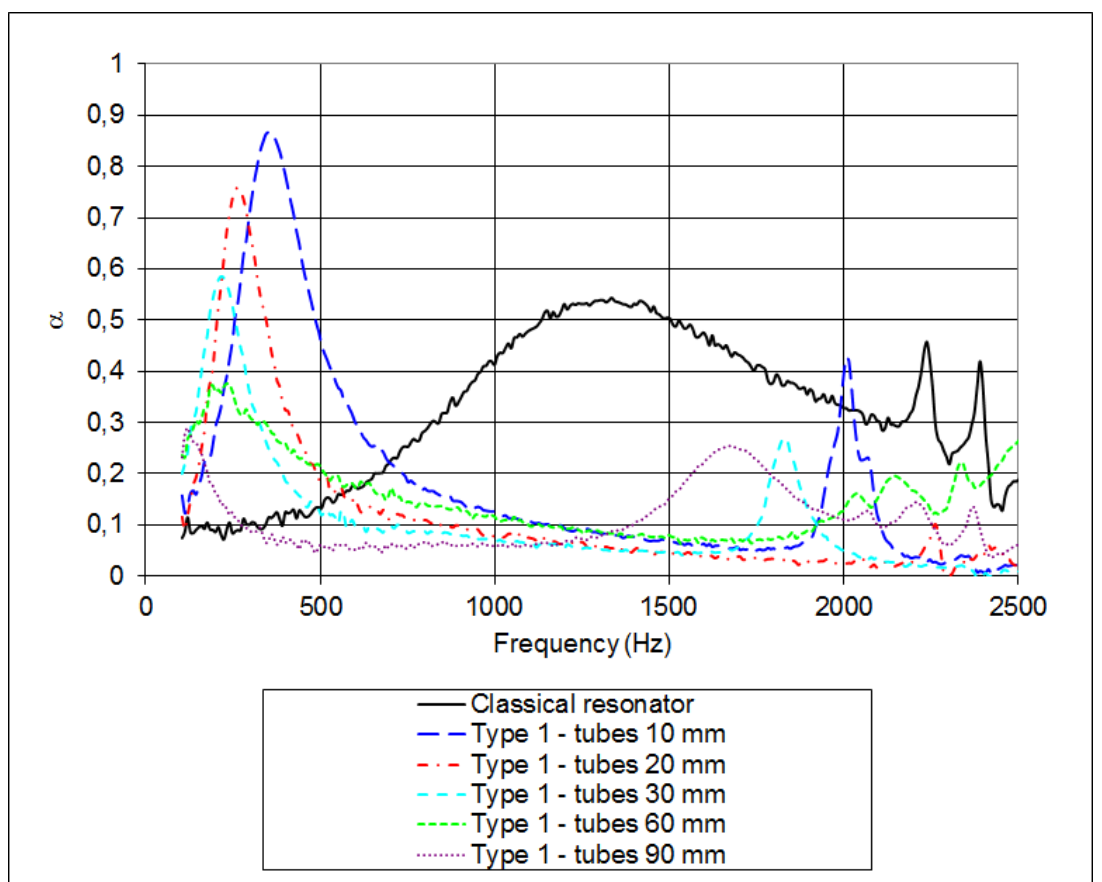

1 samples, vs. classical resonator (cf. Fig. 9).

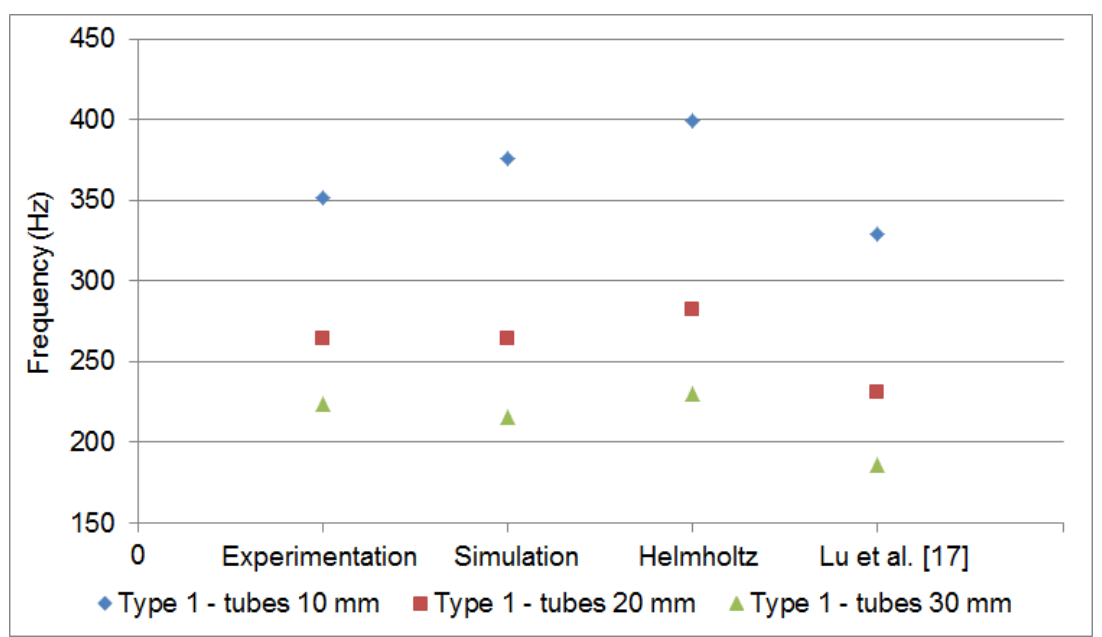

$7 \quad$ Fig. 11. Comparison of simulated and experimental frequencies of "0" reactance for 8 Type 1 samples with variable tube lengths $(10,20$ and $30 \mathrm{~mm})$ - comparison with

Helmholtz and Lu et al. [17] formulations. 
The simulation of reactance from Eq. (13) for tubes satisfying the characteristics of Table 1 but placed in front of a rigid background (Fig. 12), i.e. without a cavity ("closed end" tubes), shows that the maximum absorption is obtained at high frequencies (from $2,760 \mathrm{~Hz}$ ). It thus appears that the coupling with the surrounding cavity is predominant to generate an absorption in the low frequency range.

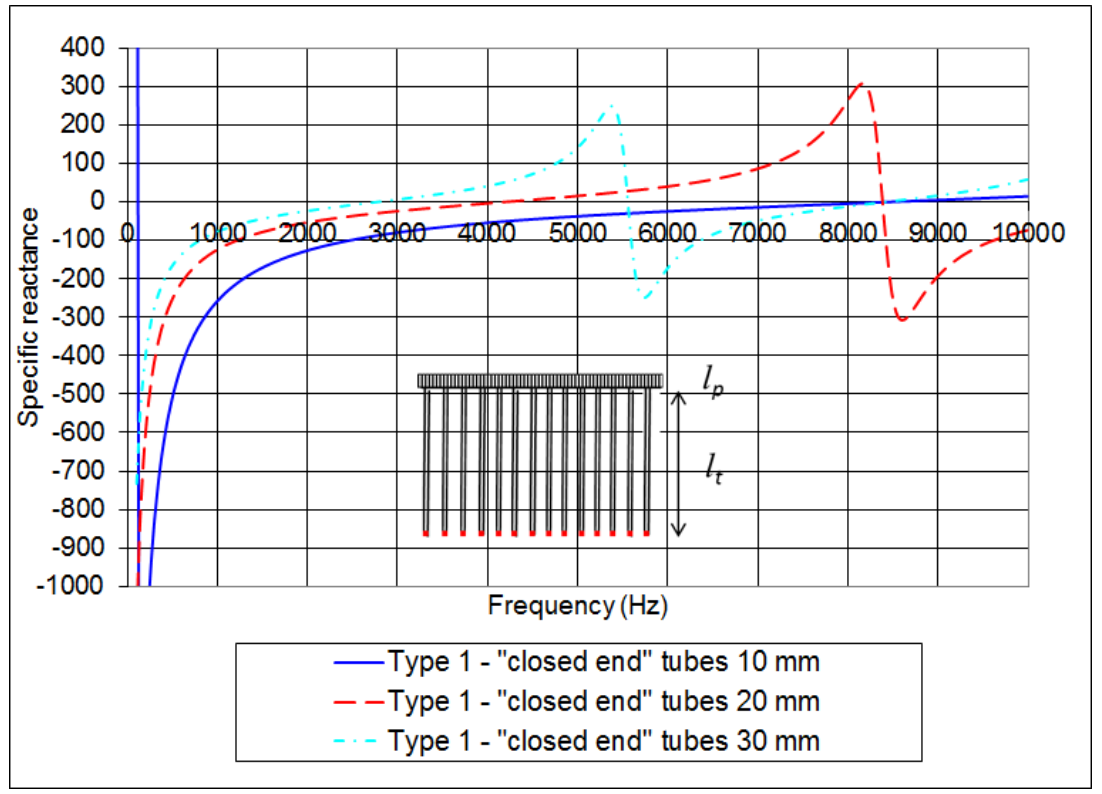

Fig. 12. Simulated reactance normalized to $\rho c$ for type 1 samples with variable "closed end" tube lengths (10, 20 and $30 \mathrm{~mm})$.

The damping relative to propagation along the tubes is also well estimated as shown on the simulated absorption coefficient values from Eq. (21) (Fig. 13). Furthermore, results would be similar to the Stinson and Champoux [26] formulation applied to long circular tubes.

Secondly, samples with variable tube radii, as specified in Table 2, are tested for two cavity thicknesses. Once again, the ratio $l_{t} / r_{i}$ is much higher than for a classical resonator.

Table 2

Characteristics of type 2 samples.

\begin{tabular}{ccccrrr}
\hline $\begin{array}{c}l_{p} \\
(\mathrm{~mm})\end{array}$ & $\begin{array}{c}\sigma_{p} \\
(\%)\end{array}$ & $\begin{array}{c}r_{i} \\
(\mathrm{~mm})\end{array}$ & $\begin{array}{c}r_{o} \\
(\mathrm{~mm})\end{array}$ & $\begin{array}{c}l_{t} \\
(\mathrm{~mm})\end{array}$ & $\begin{array}{c}h \\
(\mathrm{~mm})\end{array}$ & $l_{t} / r_{i}$ \\
\hline 1 & 3.7 & 0.6 & 0.8 & 15 & 20 & 25 \\
& 3.9 & 1.0 & 1.2 & & 30 & 15 \\
\hline
\end{tabular}


One can note, in Fig. 14, that to reduce the radius increases the absorption in 2 the main frequency range (440 and $560 \mathrm{~Hz}$ ). Moreover, as in Fig. 10, several 3 peaks appears in medium frequency range.

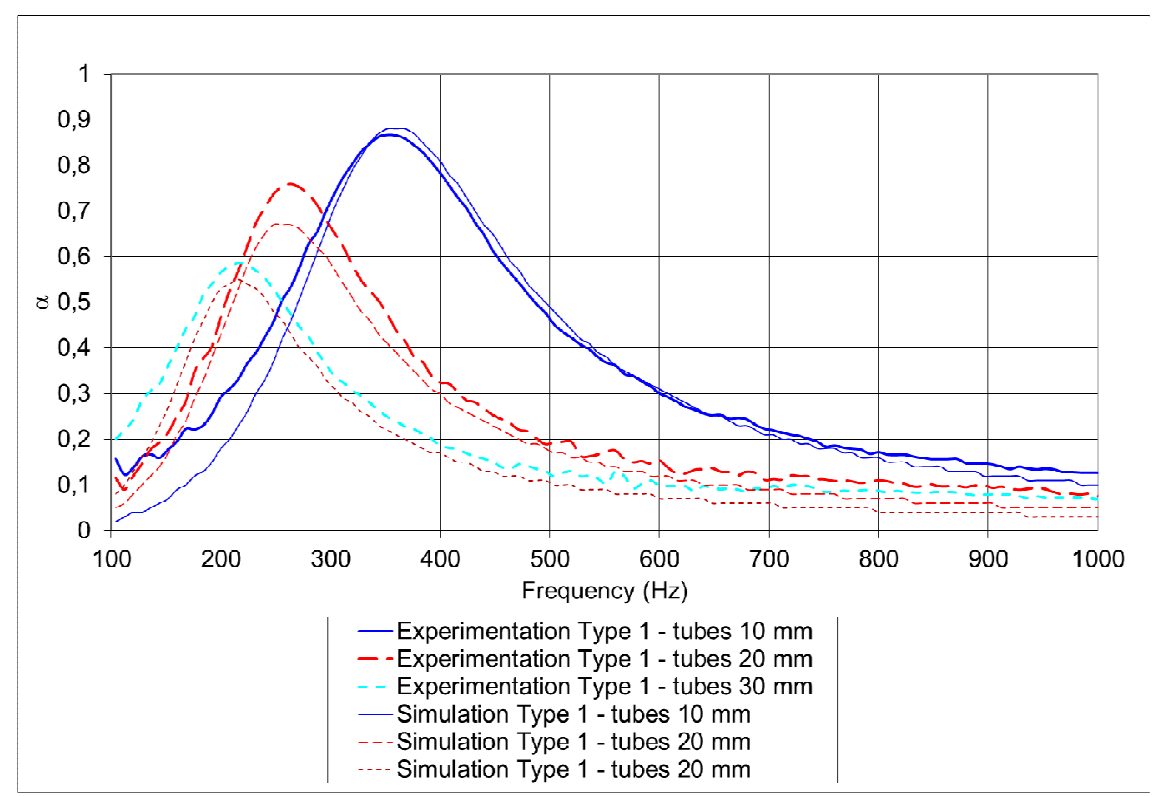

Fig. 13. Comparison of simulated and experimental absorption coefficients for type 1 samples with variable tube lengths $(10,20$ and $30 \mathrm{~mm})$.

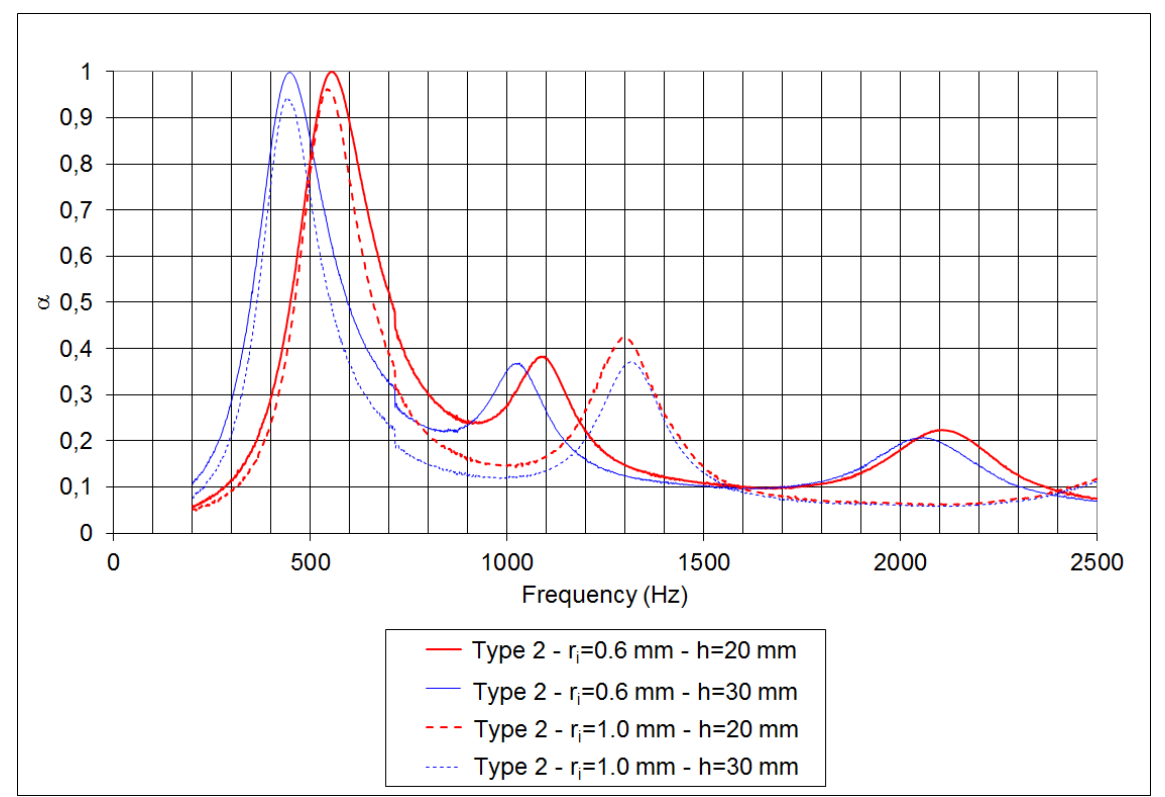

Fig. 14. Effect of tubes of variable internal radius $(0.6$ and $1 \mathrm{~mm})$ and cavity thickness (20 and $30 \mathrm{~mm}$ ) on absorption coefficient for type 2 samples (experimentation). 
The radius effect is confirmed by following simulations of the absorption coefficient (Fig. 15) even if differences appear higher. On the other hand, other regions of absorption are not predicted. One might suspect the influence of a residual vibration applied to tubes but preliminary FEM simulations of structural resonance with tube elasticity have not been conclusive.

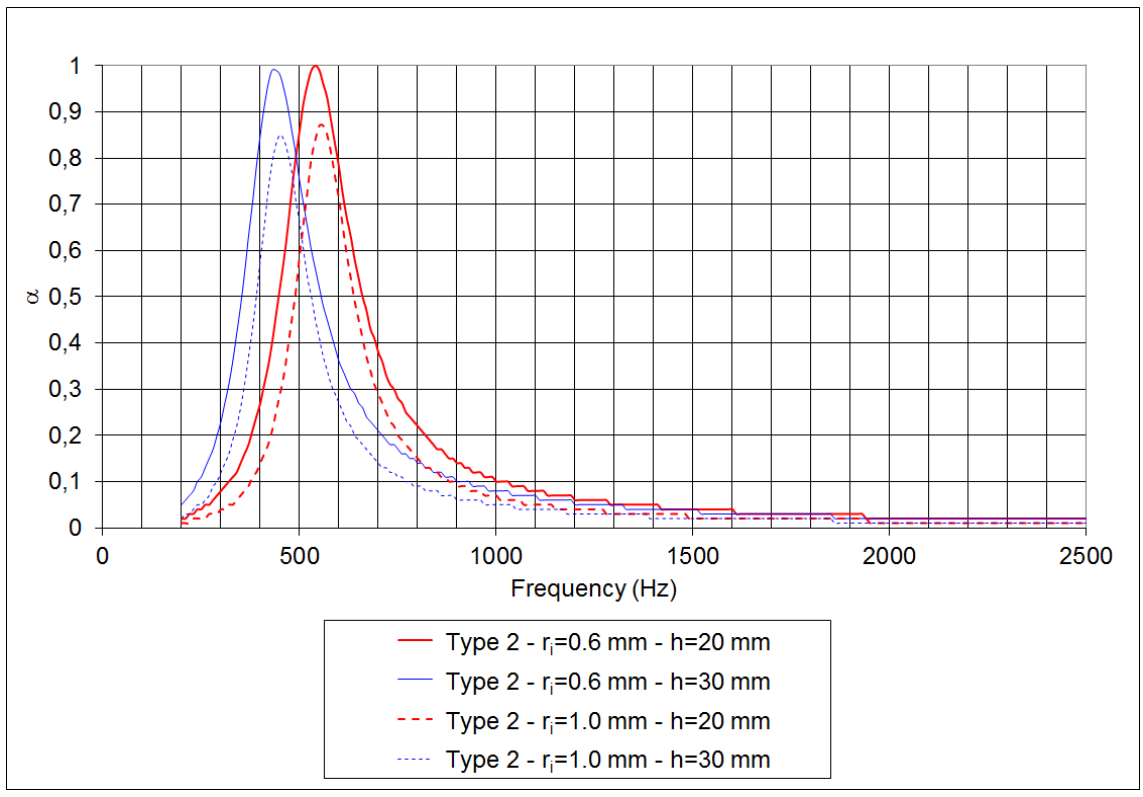

Fig. 15. Effect of tubes of variable internal radius $(0.6$ and $1 \mathrm{~mm})$ and cavity thickness (20 and $30 \mathrm{~mm}$ ) on absorption coefficients for type 2 samples (simulation).

\subsection{Type 3 Resonators}

\subsubsection{Tests in impedance tube}

Fig. 16 and 17 show a comparison of simulated and measured absorption coefficients in an impedance tube for 2 configurations of a resonator defined by the following characteristics:

- "Mono tubes" configuration : (so-called) long tubes (tube "1") or (so-called) short tubes (tube " 2 " cf. Fig. 4) with a length ratio of 3.

Other characteristics are identical for these 2 resonators, i.e. the plate porosity $\sigma_{p}$, the inner and outer tube radius, respectively $r_{i}$ and $r_{e}$, and the cavity thickness $h$. The (so-called) long tubes have a length lower than the thickness of cavity in order to use fully the effect of cavity volume.

- "Double tubes" configuration: (so-called) long and short tubes introduced simultaneously (Fig. 5). In this case, the total porosity is doubled. 
Two samples are tested:

- with a partition, for which tubes " 1 " and " 2 " are in different cavities,

- without a partition, for which tubes " 1 " and " 2 " are in the same cavity.

One can notice, for "mono tubes" (Fig. 16), an high absorption with an equivalent level regardless the frequency of interest and a coherence between experiments and simulations (from Eq. (13)). On the other hand, the frequency range can shift significantly.

The simultaneous use of two tube lengths ("double tubes" configuration) in a same cavity increases the medium frequency of absorption (Fig. 17), without extending the frequency range, which is not interesting for target applications. 13 However if the two lengths of tube are located in two different cells, there are 14 two frequency area of high absorption around the frequency range without 15 partition. Thus, a distribution with several lengths of tube in independent cells 16 can allow for an extension of the frequency range, as with two or three degrees 17 of freedom liners, but with a low cavity thickness. Moreover, it can be observed 18 that simulations (with Eq. (14) or Eq. (19)) are representative of physical 19 phenomena, regardless the tested configurations.

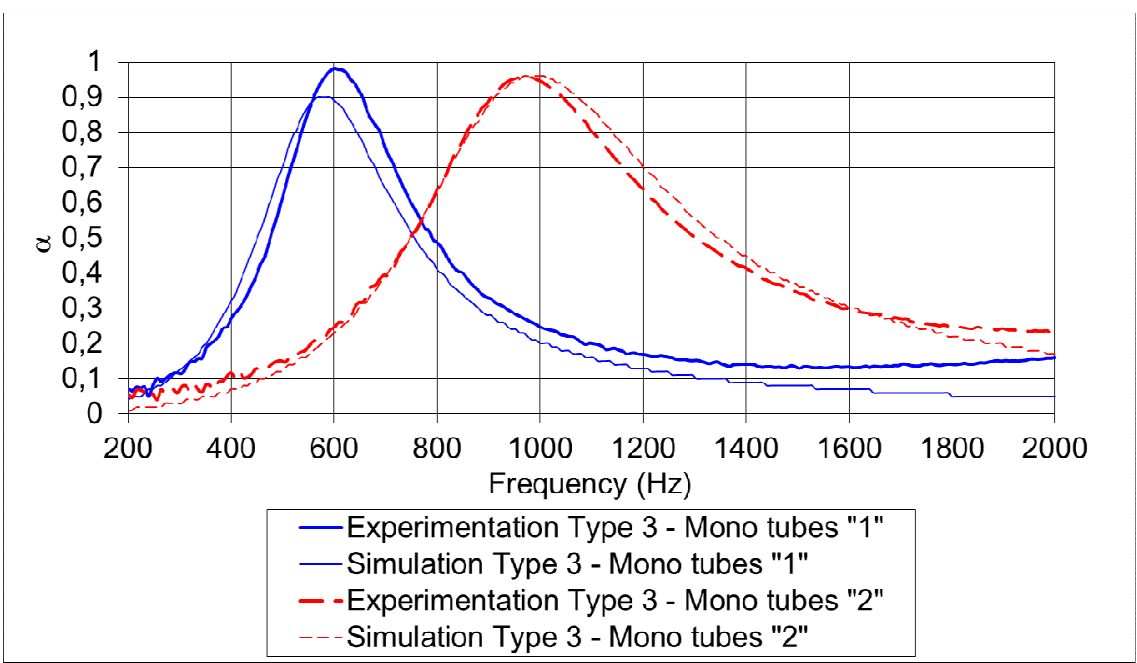

$21 \quad$ Fig. 16. Comparison of simulated and experimental absorption coefficients for type 3 samples - "mono tubes" configuration. 


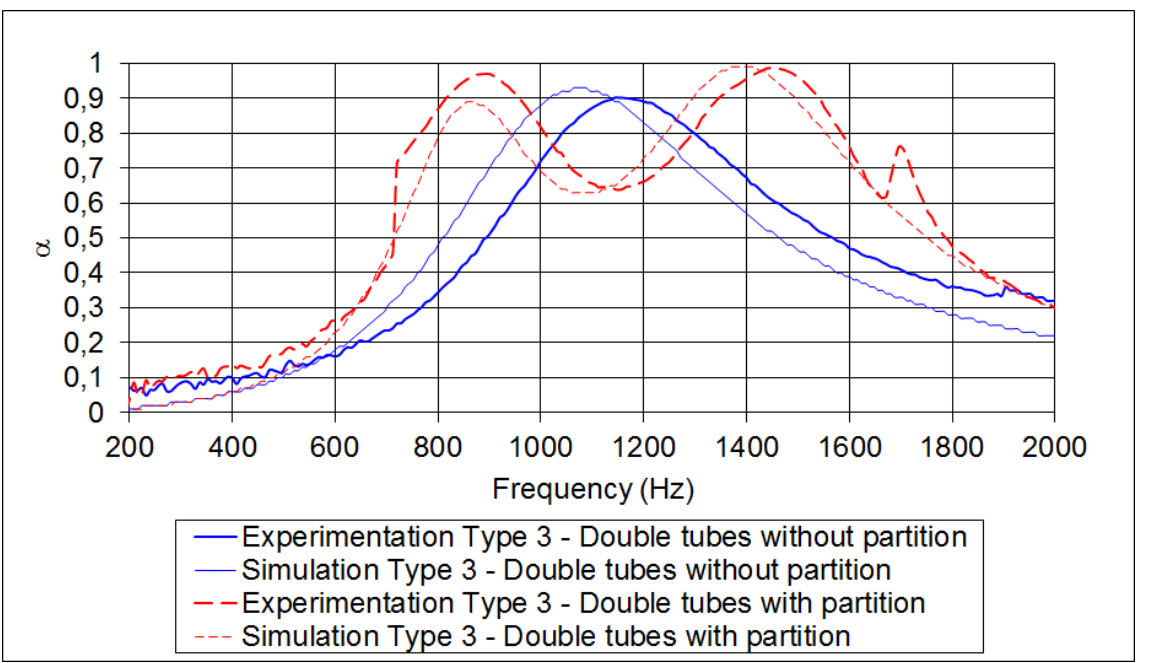

Fig. 17. Comparison of simulated and experimental absorption coefficients for type 3 samples - "double tubes" configurations without and with a partition (cf. Fig. 7).

\subsubsection{Tests in an aeroacoustic bench}

Finally, a sample of "mono tubes" with (so-called) short tubes has been manufactured to be tested with grazing flow in the aeroacoustic test bench B2A (Fig. 18).

The Aero-Thermo-Acoustic test bench is specifically used to perform in-duct flow Laser Doppler Velocimetry (LDV) or pressure measurements along an acoustic liner in presence of a grazing flow [27]. Two loudspeakers can generate plane waves, up to $3,000 \mathrm{~Hz}$, in the wind tunnel (cross-section of $50 \times 50 \mathrm{~mm}^{2}$ ) with a turbulent flow (maximum bulk Mach number $=0.5$ ). The testing cell can contain, in its lower part, a sample of material to be studied $\left(30 \times 150 \mathrm{~mm}^{2}\right.$ or 50 $14 \times 150 \mathrm{~mm}^{2}$ ). In the present case, the acoustic pressure field is acquired upstream, 15 in front of and downstream of the sample, on the opposite wall (Fig. 19).

16

17

18

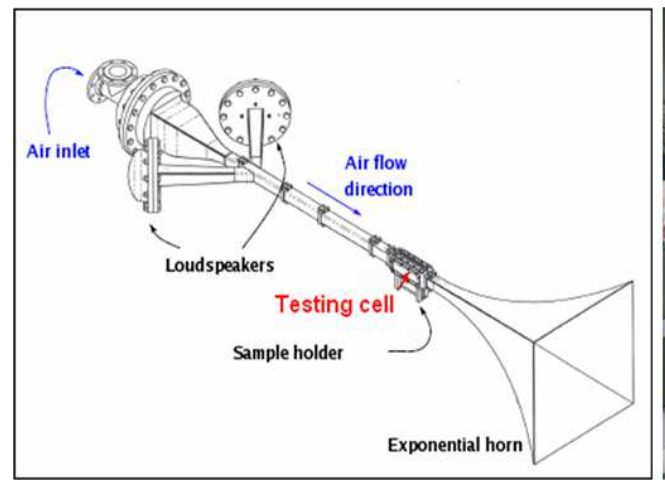

(a)

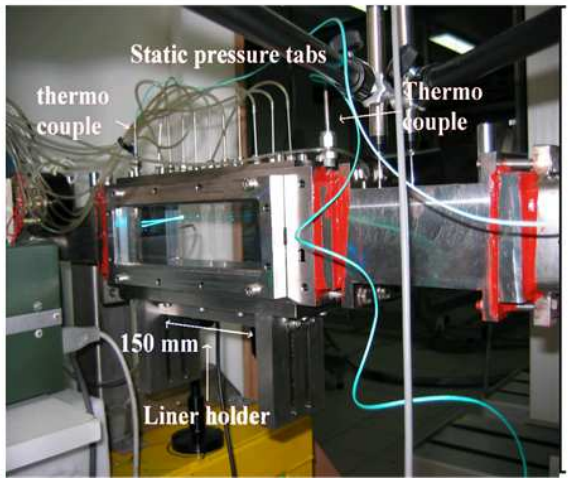

(b)

Fig. 18. Aero-acoustic bench B2A (a) with testing cell (b). 


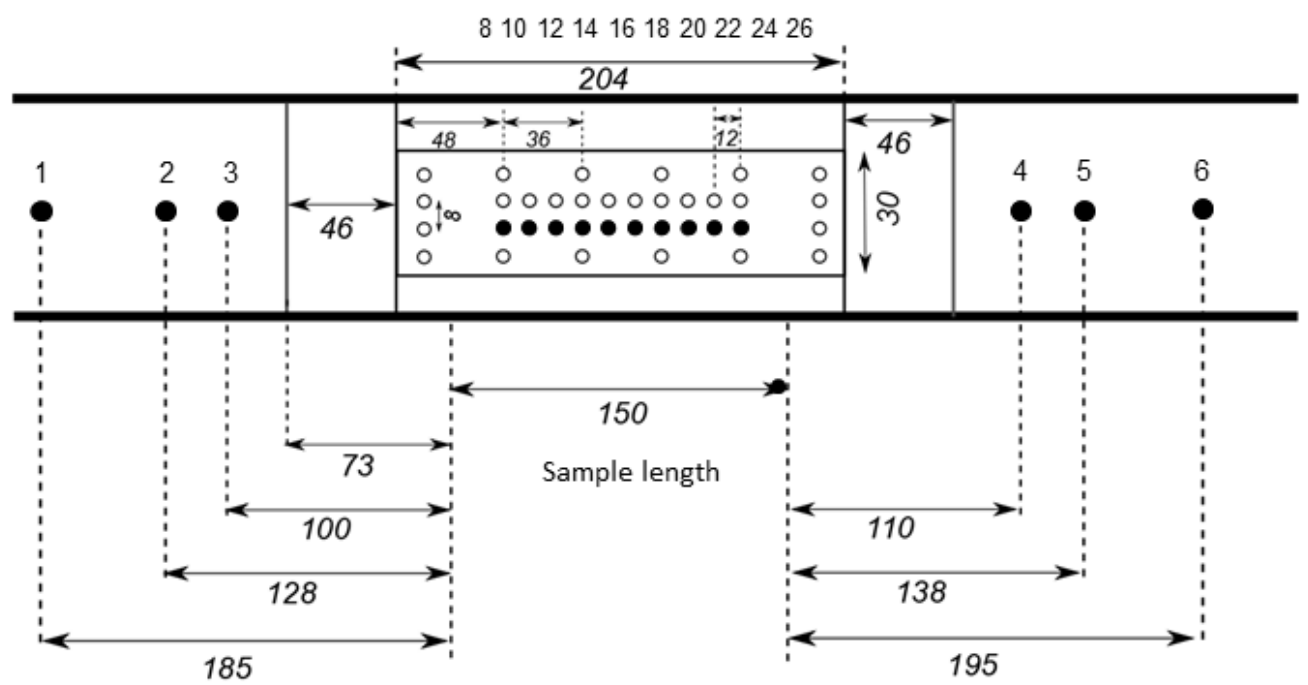

Fig. 19. Location of microphones on the upper wall of testing cell (filled circles).

Computations rely on the resolution of the 2D linearized Euler equations in the harmonic domain, spatially discretized by a discontinuous Galerkin scheme [28], which presents advantageous properties. First, it is weakly dispersive and dissipative. Additionally, boundary conditions are imposed through fluxes, which is particularly robust and straightforward.

A measurement of the acoustic transmission loss is first led without grazing flow, by separation of upstream and downstream waves with two pairs of microphones located on both sides of the test cell (Fig. 20).

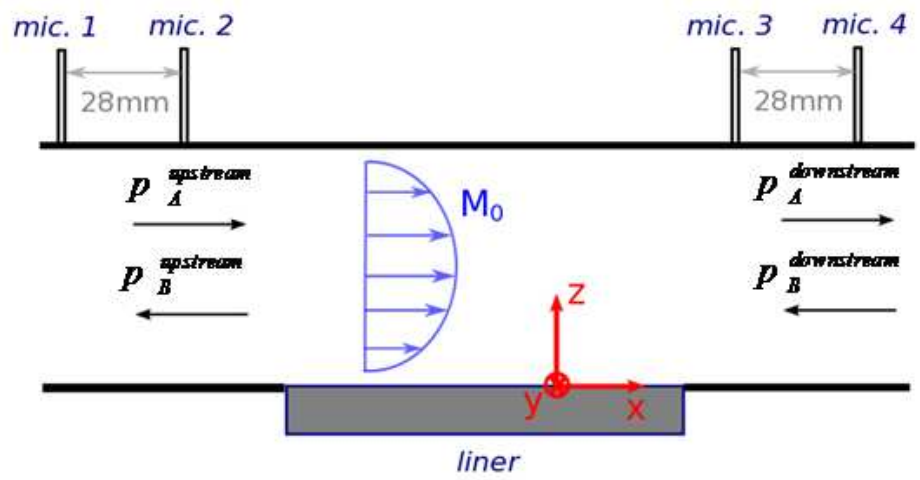

Fig. 20. Location of upstream and downstream microphones for separation of upstream and downstream waves, on either side of the liner. 
1 It appears on Fig. 21 that the maximum of Transmission Loss is reached 2 around $1,000 \mathrm{~Hz}$, the frequency of maximum absorption observed in the 3 impedance tube (cf. Fig. 15).

4

5

6

7

8

9

10

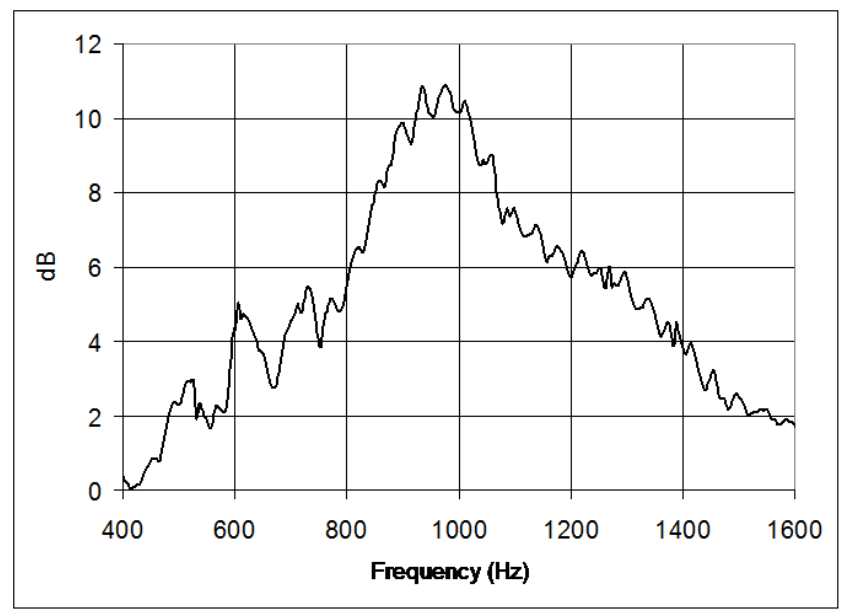

Fig. 21. Transmission Loss (dB) for a type 3 sample - "mono tubes" configuration (so-called) short tubes (tube "2"), without grazing flow.

The Fig. 22 then shows simulated and measured pressure fields at 1,000 Hz, with a grazing flow at bulk Mach number 0.3. The impedance deduced by direct simulation and comparison with measured data in B2A is close to values measured in the impedance tube (so without flow) (Fig. 23). 


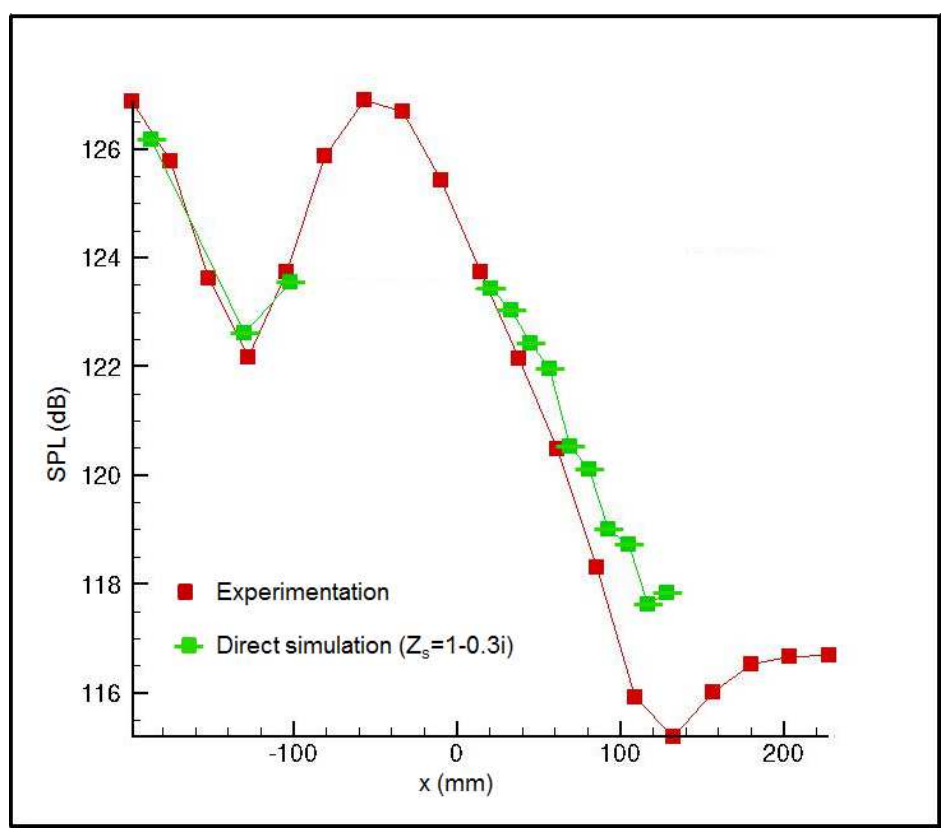

Fig. 22. Comparison of simulated and experimental pressure fields $(\mathrm{dB})$ along the testing cell for a type 3 sample - "mono tubes" configuration - (so-called) short tubes (tube "2"), at $1,000 \mathrm{~Hz}$ and bulk Mach number 0.3.

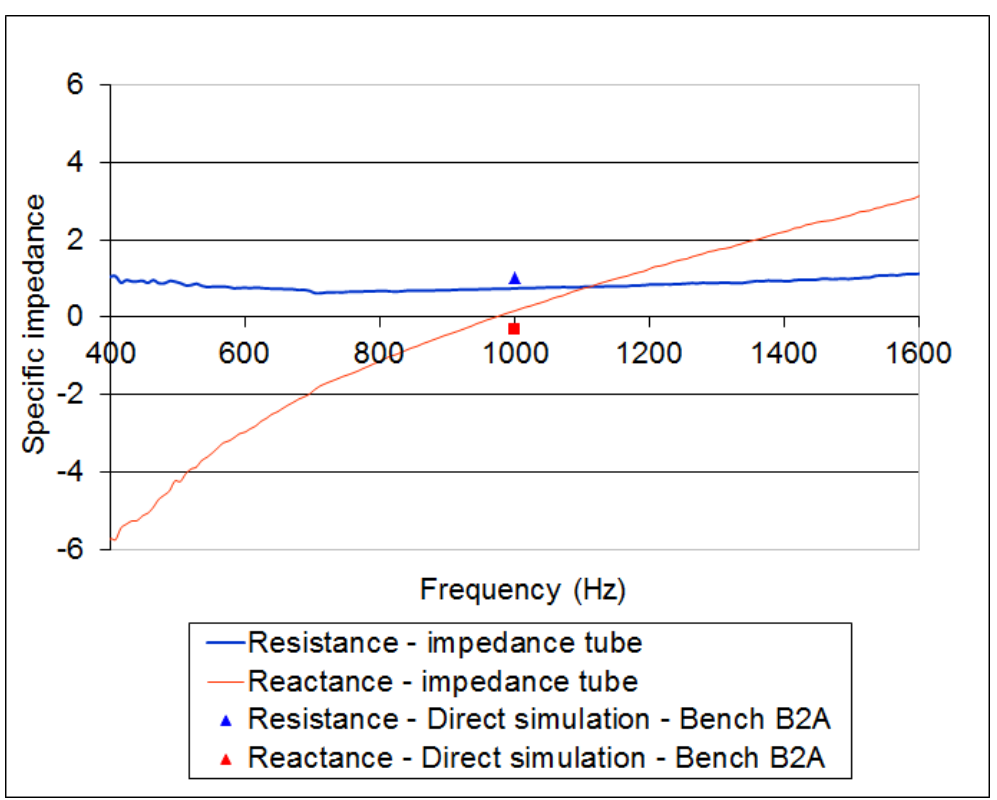

6 Fig. 23. Impedance normalized to $\rho c$ deduced from tests in B2A at bulk Mach number 70.3 and in impedance tube, for a type 3 sample - "mono tubes" configuration - so-called 8 short tubes (tube “2”). 
These results confirm the linear behavior of LEONAR resonator vs. grazing flow, contrary to a classical resonator with the same porosity. Nevertheless, complementaty tests must be conducted (by impedance eduction [28] or by micro- LDV field [29]), in particular with grazing flow for other ratios $l_{t} / r_{i}$, to confirm the absence of acoustic vortices.

\section{Conclusions}

Experimental and theoretical studies have shown that the introduction of tubes in a cavity of a conventional resonator generates a significant shift in the frequency range of absorption towards lower frequencies, due to a prolongation of the air column length provided by the presence of tubes. In this case, the thickness can reach a value lower than $\lambda / 30$.

Despite the simplicity of the theoretical approach, the impedance can be precisely determined with parallel transfer matrices of tubes coupled to the cavity. Nevertheless, the formulation must take into account the specificity of long tubes (the "thin" plate assumption is not valid anymore).

It appears, through tests in an impedance tube, that the impedance is practically independent of sound pressure level if $l_{t} / r_{i} \geq 15$. Moreover, a test led for one type or resonator in an aeroacoustic bench has shown that a grazing flow at a bulk Mach number of 0.3 has little impact on the impedance value, which is an advantage for an aeronautic application. These first results allow considering these resonators with linear behavior as an alternative to classical resonators.

Finally, industrial requirements of robustness and cleaning for aeronautical liners must be satisfied similar to these of classical resonators.

\section{Acknowledgements}

The research presented in this paper was led as part of the French project ANR MANSART (Matériaux sANdwicheS ARchiTecturés).

Many thanks for the manufacturing achieved by V. Fascio (ATECA).

\section{References}

[1] A. Cummings, Acoustic Nonlinearities And Power Losses At Orifices, AIAA J. 22 (1984) 786-792.

[2] C.K.W. Tam, K.A. Kurbatskii, K.K. Ahuja, R.J. Gaeta, A Numerical and Experimental Investigation of the Dissipation Mechanisms of Resonant Acoustic Liners, in: Proc. $7^{\text {th }}$ AIAA/CEAS Aeroacoustics Conference, Maastricht, 2001.

[3] U. Ingard, S. Labate, Acoustic Circulation Effects and the Nonlinear Impedance of Orifices, J. Acoust. Soc.Am. 22 (1950), 211-219.

[4] R.J. Gaeta, and K.K. Ahuja, Effect of Orifice Shape on Acoustic Impedance, in: Proc. 39th AIAA Aerospace Sciences Meeting \& Exhibit, Reno, 2001.

[5] A.L. Goldman, R.L. Panton, Measurement of the acoustic impedance of an orifice under a turbulent boundary layer, J. Acoust. Soc.Am. 60 (1976), 1397-1404. 
[6] J-M. Roche, L. Leylekian, F. Vuillot, 2D-axisymmetric and 3D computational study of the acoustic absorption of resonant liners, in: Proc. Internoise 2008, Shanghai, 2008.

[7] V. Chandrasekharan, M. Sheplak, L. Cattafesta, Experimental Study of Acoustic Impedance of MEMS-Based Micro-Perforated Liners, in: Proc. $12^{\text {th }}$ AIAA/CEAS Aeroacoustics Conference, Cambridge, 2006.

[8] H. Boden, Y. Guo, H.B. Tozun, Investigation of nonlinear acoustic for perforates, in: Proc. $13^{\text {th }}$ International Congress on Sound and Vibration, Vienna, 2006.

[9] H. Boden, Acoustic Characterisation of Perforates using Non-linear System Identification Techniques, in: Proc. $13^{\text {th }}$ AIAA/CEAS Aeroacoustics Conference, Roma, 2007.

[10] C. Malmary, S. Carbonne, Y. Auregan, V. Pagneux, Acoustic Impedance Measurement with Grazing Flow, in: Proc. $7^{\text {th }}$ AIAA/CEAS Aeroacoustics Conference, Maastricht, 2001.

[11] C. Heuwinkel, L. Enghardt, I. Rohle, Experimental investigation of the acoustic damping of perforated liners with bias flow, in: Proc. $13^{\text {th }}$ AIAA/CEAS Aeroacoustics Conference, Roma, 2007.

[12] M.G. Jones, M.B. Tracy, W.R. Watson, T.L. Parrott, Effects of liner geometry on acoustic impedance, in: Proc. $8^{\text {th }}$ AIAA/CEAS Aeroacoustics Conference and Exhibit, Breckenridge, Colorado, 2002.

[13] T. Dupont, P. Leclaire, O. Sicot, X. L. Gong, R. Panneton, Acoustic properties of air-saturated porous materials containing dead-end porosity, J. App. Physics 110(9) 2011, 094903-094903.

[14] P. Leclaire, O. Umnova, T. Dupont, R. Panneton, Acoustical properties of air-saturated porous material with periodically distributed dead-end pores, J. Acoust. Soc.Am. 137(4) (2015), 1772-1782.

[15] A. S. Hersh, B. Walker, J. W. Celano, Effect Of Grazing Flow and SPL On Impedance Of 2-Dof Resonators, in: Proc. $8^{\text {th }}$ AIAA/CEAS Aeroacoustics Conference And Exhibit, Breckenridge, 2002.

[16] S. Nakanishi, Sound absorption of Helmholtz resonator included a winding built-in neck extension, in: Proc. Internoise 2016, Hamburg, 2016.

[17] Y. Lu, H. Tang, J. Tian, H. Li, J. Yang, The Perforated Panel Resonator with Flexible Tube Bundles and Its Sound Absorption Measurements, in: Proc. Internoise 2007, Istanbul, 2007.

[18] F. Setaki, M. Tenpierik, A.van Timmeren, M. Turrin, New Sound Absorption Materials: Using Additive Manufacturing for Compact Size, Broadband Sound Absorption at Low Frequencies, in: Proc. Internoise 2016, Hamburg, 2016.

[19] F. Simon, Low frequency sound absorption of resonators with flexible tubes, in: Proc. ICA 2013, Montréal, 2013.

[20] F. Simon, Long elastic open neck acoustic resonator in flow, in: Proc. Internoise 2016, Hambourg, 2016.

[21] U. Ingard, Notes on "Sound absorption technology", Version 94-02, ISBN 0-931784-28-X, 1994.

[22] T. H. Melling, The acoustic impedance of perforates at medium and high sound pressure level, J. Sound Vib. 29(1) (1973),1-65.

[23] Standard Test Method for Impedance and Absorption of Acoustical Materials Using a Tube, Two Microphones, and a Digital Frequency Analysis System, American Society for Testing and Materials Designation, E 1050 - 90.

[24] J. Y. Chung, D. A. Blaser, Transfer function method of measuring in duct acoustic properties - I Theory II Experiment, J. Acoust. Soc.Am. 68(3) (1980), 907-921.

[25] H. Boden, M. Abom, Influence of errors on the two microphones method for measuring acoustics properties in ducts, J. Acoust. Soc.Am. 79(2) (1986), 541-549. 
1 [26] M. R. Stinson, Y. Champoux, Propagation of sound and the assignment of shape factors in model porous materials having simple pore geometries, J. Acoust. Soc.Am. 91 (2) 1992, 685-695.

[27] A. Minotti, F. Simon, F. Gantié, Characterization of an acoustic liner by means of Laser Doppler Velocimetry in a subsonic flow, Aerosp. Sci. Technol. (2007), doi:10.1016/j.ast.2007.09.007.

[28] J. Primus, E. Piot, F. Simon, An adjoint-based method for liner impedance eduction: validation and numerical investigation, J. Sound Vib. 332 (2013) 58-75.

[29] E. Piot, F. Micheli, F. Simon, LDA investigation of the acoustic field above a perforated liner with grazing and bias flow, in: Proc. Internoise 2009, Ottawa, 2009. 
$1 \quad$ Fig. 1. Illustration of resonator with an upper perforated plate (thickness $l_{p}$ ) connected

2 to hollow flexible tubes inserted in a cavity (thickness $h$ ).

3 Fig. 2. Type 1 samples with a perforated plate connected to Teflon tubes of variable 4 lengths, to place above a cavity.

5 Fig. 3. Type 2 samples with a perforated plate connected to PMMA tubes of variable 6 radius (a) and (b), to place above a cavity.

7 Fig. 4. Type 3 samples with a perforated plate connected to tubes (a) to place above a 8 cavity ended by a rigid wall (b).

9 Fig. 5. Type 3 samples with a perforated plate connected to tubes of 2 variable lengths, 10 partitioned (a) or not (b), to place above a cavity.

11 Fig. 6. Physical configuration (a) with an upper perforated plate connected to hollow 12 tubes (length $l_{t}$ ) inserted in a cavity (thickness $h$ ) - Simulated configuration (b) with an 13 equivalent thick perforated plate (thickness $l_{t}$ ) inserted in a cavity (thickness $h$ ).

Fig. 7. Physical configurations with an upper perforated plate connected to two types of 16 hollow tubes (lengths $l_{t 1}$ and $l_{t 2}$ ) inserted in two cavities (a) or in the same cavity (b).

17 Fig. 8. Effect of Soud Pressure Level (dB) on absorption coefficient for a type 1 sample 18 with $l_{t}=20 \mathrm{~mm}$ and $h=35.9 \mathrm{~mm}$.

19 Fig. 9. Effect of Soud Pressure Level $(\mathrm{dB})$ on absorption coefficient for a classical 20 resonator with a thin perforated plate $\left(\sigma_{p}=4.7 \%, r_{e}=0.55 \mathrm{~mm}, l_{p}=1 \mathrm{~mm}\right)$ above a cavity 21 of $35.9 \mathrm{~mm}$ thickness.

22 Fig. 10. Effect of tube length (between 10 to $90 \mathrm{~mm}$ ) on absorption coefficient for type 231 samples, vs. classical resonator (cf. Fig. 9).

24 Fig. 11. Comparison of simulated and experimental frequencies of " 0 " reactance for 25 Type 1 samples with variable tube lengths $(10,20$ and $30 \mathrm{~mm})$ - comparison with 26 Helmholtz and Lu et al. [17] formulations.

27 Fig. 12. Simulated reactance normalized to $\rho c$ for type 1 samples with variable "closed 28 end" tube lengths $(10,20$ and $30 \mathrm{~mm})$.

29 Fig. 13. Comparison of simulated and experimental absorption coefficients for type 1 30 samples with variable tube lengths (10, 20 and $30 \mathrm{~mm})$.

31 Fig. 14. Effect of tubes of variable internal radius $(0.6$ and $1 \mathrm{~mm})$ and cavity thickness 32 (20 and $30 \mathrm{~mm}$ ) on absorption coefficient for type 2 samples (experimentation).

33 Fig. 15. Effect of tubes of variable internal radius $(0.6$ and $1 \mathrm{~mm})$ and cavity thickness 34 (20 and $30 \mathrm{~mm}$ ) on absorption coefficient for type 2 samples (simulation). 
1 Fig. 16. Comparison of simulated and experimental absorption coefficients for type 3

2 samples - configuration "mono tubes".

3 Fig. 17. Comparison of simulated and experimental absorption coefficients for type 3 4 samples - configuration "double tubes" without and with partition (cf. Fig. 7).

$5 \quad$ Fig. 18. Aero-acoustic bench B2A (a) with testing cell (b).

6 Fig. 19. Location of microphones on the upper wall of testing cell (filled circles).

7 Fig. 20. Location of upstream and downstream microphones for separation of upstream 8 and downstream waves, on either side of the liner.

9 Fig. 21. Transmission Loss (dB) for a type 3 sample - configuration "mono tubes" 10 so-called short tubes (tube "2"), without grazing flow.

11 Fig. 22. Comparison of simulated and experimental pressure fields $(\mathrm{dB})$ along the 12 testing cell for a type 3 sample - configuration "mono tubes" so-called short tubes (tube 13 “2”), at 1,000 $\mathrm{Hz}$ and bulk Mach number 0.3.

14 Fig. 23. Impedance normalized to $\rho c$ deduced from tests in B2A at bulk Mach number 150.3 and in impedance tube, for a type 3 sample - configuration "mono tubes" so-called 16 short tubes (tube “2”). 